\title{
Selected Topics on Managing Complexity and Information Systems Engineering: Editorial Introduction to Issue 8 of CSIMQ
}

\author{
Peter Forbrig $^{1}$, Sergio Espana ${ }^{2}$ and Mirjana Ivanovic ${ }^{3}$ \\ ${ }^{1}$ Institute of Computer Science, University of Rostock, Albert-Einstein-Str. 22, D-18055 \\ Rostock, Germany \\ ${ }^{2}$ Department of Information and Computing Sciences, Utrecht University, Heidelberglaan 8, \\ 3584 CS Utrecht, The Netherlands \\ ${ }^{3}$ Department of Mathematics and Informatics, Faculty of Sciences, University of Novi Sad, \\ Dr. Zorana Đinđića 1, 21000 Novi Sad, Serbia \\ peter.forbrig@uni-rostock.de, s.espana@uu.nl,mira@dmi.uns.ac.rs
}

Software development has to cope with rapidly changing requirements. Therefore, agile approaches like SCRUM or Kanban became very popular. They are supported by iterative process models. Such iterative processes help to meet the requirements of customers and users. Business process models help to specify the activities in the application domain. These models can be specified for different purposes and from different perspectives. They are very important for developing a common ground about the application domain of all stakeholders.

Business process models greatly contribute to analyze and understand the activities of enterprises. However, it is still a challenge to cope with the complexity of systems specifications and their requirements. This issue of the journal of Complex Systems Informatics and Modeling (CSIMQ) presents papers that discuss selected topics on managing complexity and information systems engineering. The papers are extended versions of selected papers from the workshop on Continuous Requirements Engineering held at the requirements engineering conference REFSQ 2016 in Gothenburg, the workshop on Managed Complexity held at the business informatics conference BIR 2016 in Prague, and the CAiSE 2016 Forum held in Ljubljana.

The first paper provides a method for characterizing the importance of requirements. It has the title: "A Method for Software Requirement Volatility Analysis Using QFD". It is a joined work of three colleagues from Japan and Indonesia. The authors mention that it would be easier to accept the fact that requirements change rather than to try to avoid these changes. One should accept changing requirements and try to anticipate those changes. They provide the Quality Function Deployment (QFD) method to characterize the volatility of requirements. The degree of volatility of requirements is based on changes of architectural design elements that are created because of these requirements. The paper discusses a simple example and a case study.

The potential of dynamic perspectives on business processes is discussed by the second paper. It is written by two authors of the Johannes Kepler University Linz in Austria. It has the title: "Exploring the Potential of Dynamic Perspective Taking on Business Processes" and focuses on the intertwining of business process modeling with organizational development. They highlight the advantages of subject-oriented and social business process management. Based on that, new opportunities for stakeholder interaction provide novel perspectives on how to design business processes. The introduced dual-mode workflow execution engine UeberFlow allows considering the perspectives of communication among 
stakeholders and system interaction design during process runtime. It allows stakeholders to switch between modeling and execution and in this way facilitates the coupling of business process management with organizational development.

The application area for business process modeling is expanding. This fact is mentioned in the third paper that additionally argues that enterprises are faced with the fact that the same process is modeled for different purposes. The paper provides a framework to bridge the gap between business processes, business process modeling language, and business process modeling goal. It has the title: "The Formalization of the Business Process Modeling Goals" and is written by two colleagues of the Riga Technical University, Latvia.

Modeling business processes is a human intensive work even if it is supported by tools. Reuse of already specified models could be a way to reduce the efforts of human resources. The fourth paper follows this idea by discussing generic components for task models and workflow specifications. In contrast to general components these generic components do not support information hiding. Their purpose is to make the resulting model visible in detail. The paper is provided by two authors of the University of Rostock in Germany and has the title: "Managing Complexity in Activity Specifications by Separation of Concerns and Reusability". Beside reusability it discusses ways for separating concerns during modeling. A subject-oriented approach supported by task models is introduced. It provides opportunities for very precise specifications that are still readable.

Coping with complexity is very much related to metrics and measuring. The title of the fifth paper is: "Measuring Complexity of SAP systems". The two authors from the University of Economics in Prague discuss existing methodologies for handling complexity. They provide a method for measuring of ERP systems based on their research for the SAP ERP system. Additionally, they provide ideas verifying their approach.

The problem of Shadow IT is raised by the sixth paper from three authors from the University of St. Gallen in Switzerland and the Zagreb School of Economics and Management in Croatia. The title of the paper is: "Influence of Shadow IT on Innovation in Organizations". Shadow IT describes software solutions that are brought in by employees and are not officially approved by the company. The authors discuss how this phenomenon can be turned from a problem like security concerns to an opportunity of innovation.

Last but not least, this issue is closed by a paper that discusses specific problems of information systems. It focuses on creating identity federations between different electronic identities (eID) systems. The paper has three authors from two universities. These universities are the University of Lisbon in Portugal and the Graz Technical University in Austria. It has the title: "Employing Ontology-Alignment and Locality-Sensitive Hashing to Improve Attribute Interoperability in Federated eID Systems".

The editors of this special issue of CSIMQ are thankful to all authors, who submitted their papers. Our thanks also go to the reviewers who provided very helpful comments and ideas for improvement. Finally, we would like to express our appreciation to the publisher who gave us the chance to edit this issue and who helped to provide a perfect layout of all papers. We hope you enjoy reading them. 